\title{
Evaluation of Paranasal Sinus Lesions through Plain X-rays and CT-Scan - A Descriptive Study
}

\author{
Mayur V. Khandedia ${ }^{1}$, Vishalkumar H Bhardava ${ }^{2}$, Akshay Sarda ${ }^{3}$ \\ ${ }^{1}$ Associate Professor, Department of Radiology, SBKS Medical College, Piparia, Vadodara, ${ }^{2}$ Assistant professor, Department \\ of Radiology, SBKS Medical College, Piparia, Vadodara, ${ }^{3}$ Senior Resident, Department of Radiology, MVP Medical College, \\ Adgaon, Nasik, India
}

Corresponding author: Vishalkumar H Bhardava, D4, Staff Quarters, Sumandeep Vidyapeeth University, Piparia, Vadodara 391760

DOI: http://dx.doi.org/10.21276/ijcmsr.2019.4.1.3

How to cite this article: Mayur V. Khandedia, Vishalkumar H Bhardava, Akshay Sarda. Evaluation of paranasal sinus lesions through plain X-rays and CT-scan- a descriptive study. International Journal of Contemporary Medicine Surgery and Radiology. 2019;4(1):A9-A14.

\section{A B S T R A C T}

Introduction: X-ray PNS is the primary investigation for paranasal sinus lesions, but many times it is not sufficient to arriveat final diagnosis. Also plain radiographs do not provide information for pre-operative surgical planning. CT scan can become a useful diagnostic modality in the evaluation of PNS and an integral part of surgical planning. It can also be used to create Intra-operative road maps.

Material and methods: The study comprised of 78 patients who presented to department of radiology, Dhiraj general hospital for evaluation of paranasal sinuses and were evaluated with plain X rays and CT scan of PNS.

Results: Maxillary sinus (96.3\%) was most commonly affected in inflammatory conditions with blocked OMC in $48 \%$ cases whereas frontal sinus was affected in cases of Osteoma. All the cases of malignancy involved maxillary sinus with most frequent intraorbital (70\%) extension.

Conclusion: CT scan of paranasal sinuses with axial and coronal reconstruction is pre-requisite for detailed evaluation of paranasal sinus lesions with lesion characterization and its extent best evaluated with CT scan which also provides roadmap for sinus surgeries.

Keywords: Sinusitis, Osteomeatal Complex, Antrochoanal Polyp, Wegeners Granulomatosis, Inverted Papilloma

\section{INTRODUCTION}

The paranasal sinuses are a group of air containing spaces which are hollow and located within the bones of the face and base of the skull that surrounds the nasal cavity. They derive their nomenclature from the bones that they pneumatize. They comprise the maxillary, the frontal, the ethmoid and the sphenoid sinuses. Various sinuses may follow different calendars, their origin is the same. Development of head and neck along with face, nose and paranasal sinuses takes place simultaneously in a short window span. It is the first paranasal sinus to form. It appears within few weeks of the birth and reaches adult size at puberty. Maxillary sinuses are the first paranasal sinuses to form. They appears within few weeks of the birth and reaches adult size at puberty. During the later phase, pneumatization spreads more inferiorly as the permanent teeth take their place. Pneumatization can be so extensive as to expose tooth roots with only a thin layer of soft tissue covering them. ${ }^{1,2}$

The ethmoid sinuses appear during the fifth foetal month as invaginations of the middle meatus of the nasal passages (the space underlying the middle nasal concha) and grow into the ethmoid bone. They are present at birth but small before 2 years. They reach adult size by the age of 12 years. The nasofrontal region arises from an evagination of that deep anterosuperior part of the middle meatus termed the frontal recess. The upward expansion continues, with the cell at first laying closer to the posterior table than to the anterior one before it finally rests in the cancellous bone midway between the two tables. ${ }^{3,4}$

The sphenoidal sinus begins in the 4-month of foetal life as an evagination from the posterior part of the nasal capsule into the sphenoid bone. It continues to enlarge throughout infancy and childhood. PNS lesions are common in all the age groups. Various types of diseases which affect the paranasal sinuses include congenital abnormalities, benign tumors, inflammatory and allergic conditions, complications of infection, obstruction, and traumatic lesions. These all can be evaluated with the help of X-rays, CT, and MRI (Figure no. 1 and 2).Sinusitis is a condition consisting of infection or inflammation of the paranasal sinuses, which may or may not be as a result of infection, from bacterial, fungal, viral, allergic or autoimmune issues. ${ }^{5,6}$

Newer classifications of sinusitis refer to it as rhinosinusitis, taking into account the thought that inflammation of the sinuses cannot occur without some inflammation of the nose as well (rhinitis). Sinusitis can be acute (going on less than four weeks), subacute (4-12 weeks) or chronic (going on for 
12 weeks or more). ${ }^{7}$

Majority of timesX-ray PNS is the primary investigation for paranasal sinus lesions, but in many cases it is not sufficient to arrive at final diagnosis. Also plain radiographs do not help provide information for preoperative surgical planning. In a study of review of CT of paranasal sinusitis with clinical and surgical findings, it was found that CT provides an excellent map to help the sinus surgeon operate. CT provides information about the extent of mucosal disease but this correlates poorly with symptoms, surgical findings and histopathology. 8,9

With the use of CT scanning combined with functional endoscopic sinus surgery (FESS), modern sinus surgeons can treat patients more effectively, facilitating reduced morbidity and complications. CT scan can become a useful diagnostic modality in the evaluation of the PNS and an integral part of surgical planning. It can also be used to create Intraoperative road maps and the radiological examination of choice in evaluating the PNS of a patient with sinusitis. ${ }^{10,11}$ Therefore, the main aim of the present study was to diagnose lesions affecting paranasal sinuses with high accuracy and also to evaluate the paranasal sinus lesions for their etiology which can provide roadmap for surgeons for proper planning of surgery.

\section{MATERIAL AND METHODS}

This was a prospective descriptive study which aims at following up cases of paranasal sinus lesions which were presented at Department of Radiodiagnosis, Dhiraj General Hospital, SBKS Medical college and Research Centreby using X ray PNS and CT scan (Figure no. 3). All the patients presented to Dhiraj General Hospital for the purpose of diagnosis and treatment. Total number of patients included in this study was 78 patients out of which 52 were males and 26 were females. Only those patients who gave informed written consent participated in this study. Patients referred to the radiology department for plain X-ray and/or CT scan PNS investigations, and found to have paranasal sinus lesion, were included in the present study. Already diagnosed cases of paranasal sinus lesions which needed follow up radiological investigations and are referred to our radiology department was included in the current study. Patients coming for plain $\mathrm{X}$-rays and CT scan for diseases other than paranasal sinus lesions, and are accidentally found to have paranasal sinus lesion, were also included in this study.Patients who were presented to Department of Radiology with paranasal sinus lesions in past and were cured completely were excluded from the study. Description of the materials used for this present study is as follows:

\section{X-Ray Machine : $600 \mathrm{~mA}$ Siemens $500 \mathrm{~mA}$ Siemens $300 \mathrm{~mA}$ Siemens \\ 2. CR system \\ : Kodak (agfa)}

3. CT scan Machine : Siemens emotion 16

All patients were presented with clinically suspected paranasal sinuses diseases. The selected patients were subjected to detailed history and required clinical examination and also underwent to $x$-ray PNS (water's view) and CT-PNS examination in both coronal and axial planes. X-ray for paranasal sinuses were performed by Siemens X-ray machine. Water's view examination was conducted by marking the patient's orbitomeatle line to the correct angle which was 37 degrees to the table with his/her mouth open in order to locate the ethmoid and sphenoid sinuses; the central ray was kept perpendicular to the subject's head exit at acanthion, at a source image distance of $100 \mathrm{~cm}$. In order to prevent unnecessary exposure to radiation, the collimated $\mathrm{f}$ field was limited to a range covering just the four sinuses. Exposure factor was kept at $75 \mathrm{Kvp}$ and $20 \mathrm{mAs}$.

\section{RESULTS}

This study comprised of 52 males and 26 females, between age groups of 00 - 90 years. The peak incidence was observed in the age group of 21- 40 years, which comprised 39 (50\%) of patients. Out of both the genders and all age groups, majority of the males in the age group 21-30 years were found to have

\begin{tabular}{|l|c|c|c|c|}
\hline Sr. & Age Group & \multicolumn{2}{|c|}{ No. Of Cases } & \multirow{2}{*}{ Total (\%) } \\
\cline { 3 - 4 } No. & (years) & Male & Female & \\
\hline 1. & $00-10$ & 1 & 1 & $2(2.6)$ \\
\hline 2. & $11-20$ & 7 & 2 & $9(11.5)$ \\
\hline 3. & $21-30$ & 19 & 3 & $22(28.2)$ \\
\hline 4. & $31-40$ & 8 & 9 & $17(21.8)$ \\
\hline 5. & $41-50$ & 7 & 5 & $12(15.4)$ \\
\hline 6. & $51-60$ & 8 & 4 & $12(15.4)$ \\
\hline 7. & $61-70$ & 0 & 1 & $1(1.3)$ \\
\hline 8. & $>70$ & 2 & 1 & $3(3.8)$ \\
\hline Total & & 52 & 26 & $78(100)$ \\
\hline
\end{tabular}

Table-1: Shows the distribution of patients according to the age group and gender

\begin{tabular}{|l|c|c|c|}
\hline Disease & Male & Female & Total \\
\hline Inflammatory & 30 & 21 & 51 \\
\hline Trauma & 08 & - & 08 \\
\hline Epithelial & 08 & 02 & 10 \\
\hline Soft tissue & 03 & - & 03 \\
\hline Osseous & 02 & 03 & 05 \\
\hline Odontogenic & 01 & - & 01 \\
\hline TOTAL & $52(66.7 \%)$ & $26(33.3 \%)$ & $78(100 \%)$ \\
\hline
\end{tabular}

Table-2: Shows the distribution of disease among patients based on gender

\begin{tabular}{|l|c|}
\hline Type of Disease & Perecntage (\%) \\
\hline Congenital & $00(00)$ \\
\hline Inflammatory & $51(65.4)$ \\
\hline Trauma & $08(10.3)$ \\
\hline Epithelial & $10(12.8)$ \\
\hline Salivary gland & $00(00)$ \\
\hline Neuroectodermal Neural & $00(00)$ \\
\hline Soft tissue & $03(3.8)$ \\
\hline Osseous & $05(6.4)$ \\
\hline Odontogenic & $01(1.3)$ \\
\hline Haemopoietic & $00(1.6)$ \\
\hline Metastatic & $00(00)$ \\
\hline Table-3: Shows the distribution of final radiological diagnosis \\
\multicolumn{2}{|c|}{ of the lesions among patients } \\
\hline
\end{tabular}




\begin{tabular}{|l|c|c|c|c|c|c|c|c|c|}
\hline Disease & $\mathbf{0 0 - 1 0}$ & $\mathbf{1 1 - 2 0}$ & $\mathbf{2 1 - 3 0}$ & $\mathbf{3 1 - 4 0}$ & $\mathbf{4 1 - 5 0}$ & $\mathbf{5 1 - 6 0}$ & $\mathbf{6 1 - 7 0}$ & $\mathbf{7 7 0}$ & Total \\
\hline Inflammatory & 02 & 06 & 14 & 13 & 08 & 06 & - & 02 & 51 \\
\hline Trauma & - & 02 & 03 & 01 & - & 02 & - & - & 08 \\
\hline Epithelial & - & - & 02 & 01 & 02 & 03 & 01 & 01 & 10 \\
\hline Soft tissue & - & 01 & 01 & - & 01 & - & - & - & 03 \\
\hline Osseous & - & - & 01 & 02 & 01 & 01 & - & - & 05 \\
\hline Odontogenic & - & - & 01 & - & - & - & - & - & 01 \\
\hline Total & 02 & 09 & 22 & 17 & 12 & 12 & 01 & 03 & 78 \\
\hline \multicolumn{7}{|r|}{ Table-4: Shows the distribution of disease profile among various age groups } \\
\hline
\end{tabular}

\begin{tabular}{|l|c|c|c|c|c|c|c|}
\hline & Inflammatory & Trauma & Epithelial & Soft tissue & Osseus & Odontogenic & Total (\%) \\
\hline Right Maxillary & 12 & 02 & 05 & - & 01 & - & $20(25.6)$ \\
\hline Left Maxillary & 17 & 03 & 05 & 01 & - & 01 & $27(34.6)$ \\
\hline BL Maxillary & 21 & 03 & - & 01 & - & - & $25(32)$ \\
\hline Ethmoid & 24 & 06 & 07 & - & 01 & - & $38(48.7)$ \\
\hline Frontal & 15 & 03 & 04 & - & 02 & - & $24(30.8)$ \\
\hline Sphenoid & 16 & - & 03 & 01 & - & - & $20(25.6)$ \\
\hline \multicolumn{7}{r|}{ Table-5: Shows the predominant sinus involved among the patients } \\
\hline
\end{tabular}

\begin{tabular}{|l|c|c|c|c|c|c|c|}
\hline & Obstruction & Discharge & Epistaxis & Pain & Swelling & Proptosis & Others \\
\hline Inflammatory & 17 & 42 & 03 & 26 & - & - & 10 \\
\hline Trauma & - & - & 07 & 05 & 08 & - & - \\
\hline Epithelial & 07 & 03 & 07 & 06 & 08 & 08 & - \\
\hline Soft tissue & 02 & 01 & 02 & 01 & 01 & - & 01 \\
\hline Osseus & - & - & - & 03 & 04 & 01 & 01 \\
\hline Odontogenic & - & - & - & 01 & 01 & - & 01 \\
\hline TOTAL (\%) & $26(33.3)$ & $46(59)$ & $19(24.4)$ & $42(53.8)$ & $22(28.2)$ & $09(11.5)$ & $13(16.7)$ \\
\hline \multicolumn{7}{|l}{ Table-6: Shows the distribution of symptoms among the patients according to the disease } \\
\hline
\end{tabular}

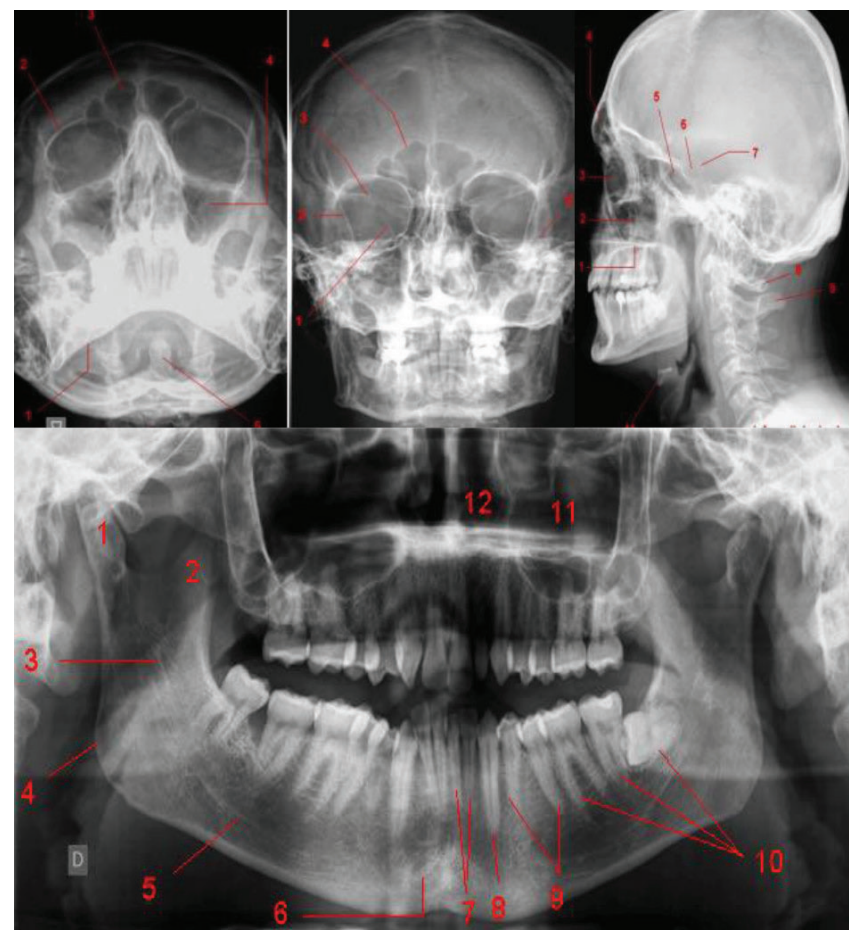

Figure-1: Shows the axial multidetector CT images

the paranasal sinuses disease i. e. 19 (24.4\%). Paranasal sinus diseases were observed least frequently in paediatric $(00-20$ years) and geriatric (> 60 years) patients (Table no. 1 ).

Male to female ratio was found to be $2: 1$ in the present
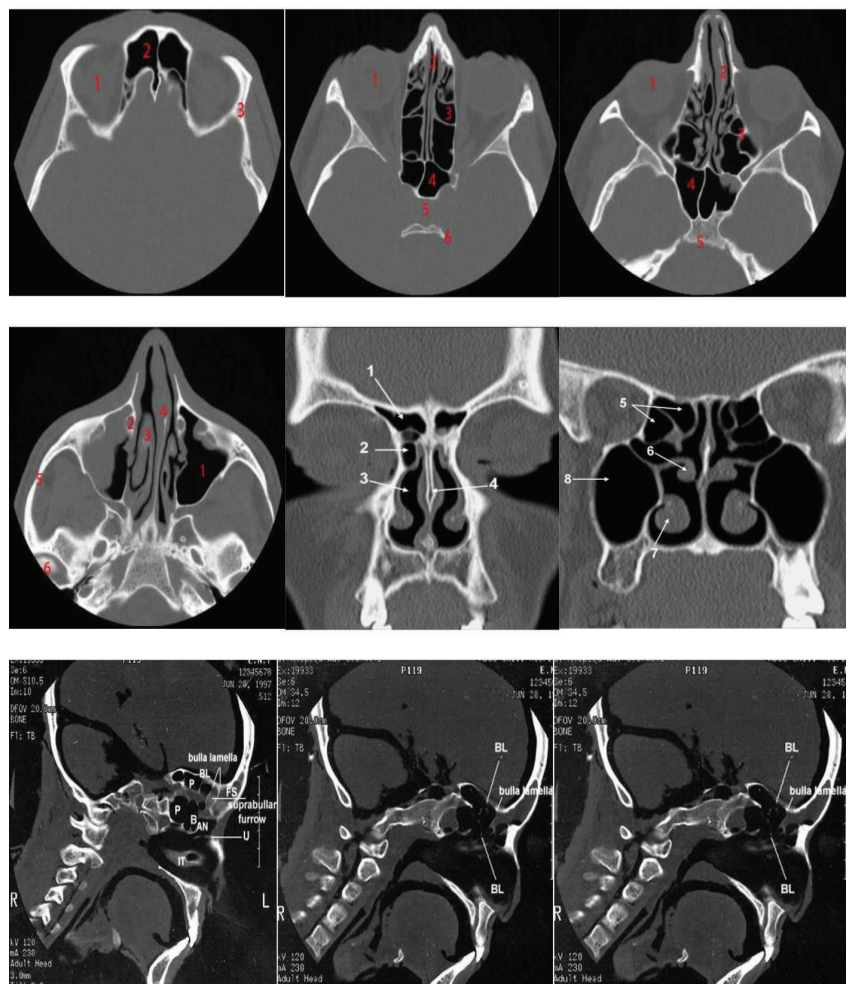

Figure-2: Shows the normal anatomy and sagittal images of paranasal sinus

study. Inflammatory conditions were seen affecting both the genders most commonly. Epithelial and soft tissue conditions 

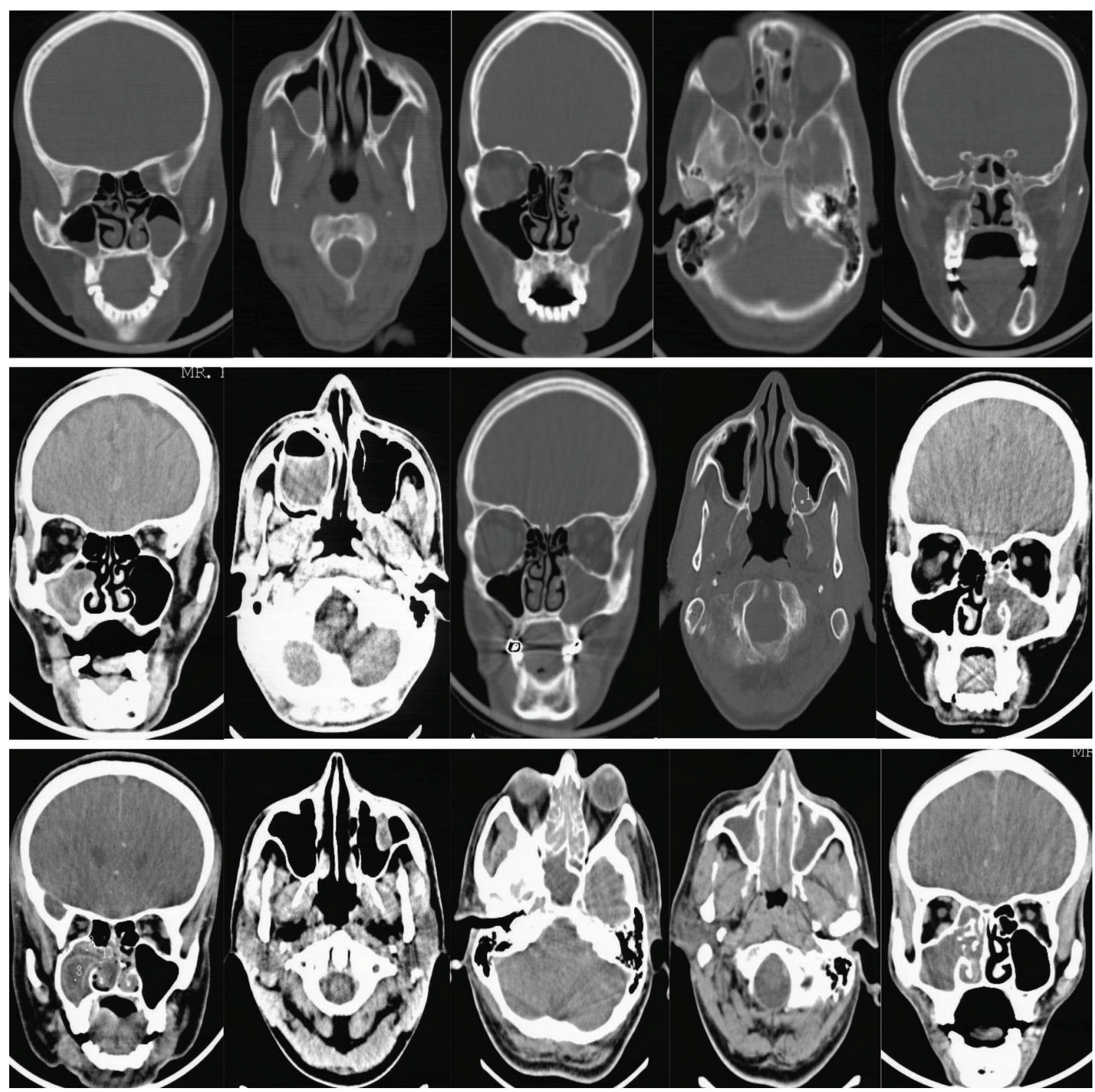

Figure-3: Shows various cases of paranasal sinus lesions including maxillary sinusitis, pansinusitis, maxillary sinus tubercular infection, mucocele, antrochoanal polyp and maxillary polyp

affected males more commonly than females, whereas more females were affected by osseous pathologies than males (Table no. 2).

It has been found that inflammatory conditions of paranasal sinuses were most common in all groups affecting $65 \%$ of patients in this study. Epithelial conditions were second common affecting only $13 \%$ of patients. Trauma to paranasal sinuses was seen among $10.3 \%$ of the patients. Osseous conditions (6.4\%) and soft tissue lesions (3.8\%) were also common, with odontogenic condition being only among 1.3\%. There was associated mastoiditis present in 3 patients (Table no. 3).

In Table no. 4, patients in age group of 21-40 years were most commonly affected by inflammatory conditions, which was also the most common condition observed in all the age groups. Trauma to paranasal sinuses was seen only in males in this study. Epithelial conditions were evenly distributed in elderly and geriatric patients, with youngest patient being 22 years old. A rare case of extra nasopharyngeal angiofibroma was seen in 17 year old male; with other soft tissue lesions were also seen affecting adults. Osseous pathologies of paranasal sinuses seem to affect patients of wide age group of 21-60 years, with nearly equal incidence in each decade. Single odontogenic pathology, secondarily involving maxillary sinus was seen in 21 year old male patient.

It has been found that maxillary sinus is affected most commonly, 72 (92.3\%) patients had involvement of maxillary sinus in this study. Left maxillary sinus was affected slightly more than bilateral involvement of maxillary sinus, followed by right maxillary sinus involvement. Inflammatory conditions affect maxillary sinus most often, bilateral involvement was seen commonly in inflammatory conditions and unilateral 
involvement in epithelial conditions. All the patients with epithelial conditions involved maxillary sinus, whereas only 1 patient with osseus pathology had maxillary involvement. Ethmoid sinus was the second most common sinus affected, predominantly in inflammatory conditions. Post traumatic hemosinus was seen commonly in ethmoid sinuses. Both the cases of osteomas were seen in frontal sinuses. Sphenoid sinus was involved predominantly in inflammatory conditions and in a case of extranasopharyngeal angiofibroma (Table no. 5) Nasal discharge (84\%) was the most common complaint in patients presenting with inflammatory conditions, followed by pain (50\%) and obstruction (33\%). In inflammatory conditions, patients with sinusitis presented with discharge and majority of patients having polyp presented with obstruction. Epistaxis was seen in only $6 \%$ of patients with inflammatory condition, while $86.5 \%, 70 \%$ and $66.7 \%$ patients with trauma, epithelial conditions and soft tissue lesion, respectively presented with epistaxis. Epithelial diseases were presented commonly with swelling, epistaxis and proptosis due to propensity to extend into nasal cavity and intraorbitally. Pain and swelling were chief complaints for patients with osseous pathologies. (Table no. 6)

\section{DISCUSSION}

In this study, most common sinus involvement was maxillary sinus (96.3\%) and this finding was consistent with study done by Gwaltney et al. Involvement of frontal, ethmoid as well as sphenoid sinuses in cases of sinusitis are comparable in both the studies. However, incidence of occlusion of OMC was less $(44.4 \%)$ in our study, as compared to Gwaltney et al study (77\%). About 26 (33.3\%) patients had normal osteomeatal complex, despite being affected by inflammatory or osseus conditions. Blocked OMC was seen in 12 (48\%) patients of sinusitis and widened OMC in 9 (40.9\%) patients of polyp. $90 \%$ of patients with epithelial condition had destruction of OMC, which was also the finding in a case of Wegener's granulomatosis, however to a less severe extent. Unilateral involvement of sinus by polyp was seen most commonly in 45.4\% patients. Antrochoanal polyp was seen least often in $18.2 \%$ patients against $45 \%$ patients in Schramm et al study. ${ }^{12,13}$

In the present study, there were 3 cases of angiofibromas. 2 patients of nasopharyngeal angiofibroma were adult males with characteristic intense post contrast enhancement on CT scan. Both the patients presented with complaints of nasal obstruction and epistaxis. There was 1 paediatric patient with a rare case of extranasopharyngeal angiofibroma with enhancing soft tissue mass in left infratemporal fossa and extension and erosion into sphenoid sinus.

Also, there was 1 male patient of 27 years age who presented with nasal obstruction, discharge and epistaxis. His chest radiograph characteristically showed multiple bilateral well defined nodular opacities, without fibrosis. Other differential diagnoses were ruled out. His CT scan of paranasal sinuses showed soft tissue mass in left maxillary sinus and nasal cavity with minimal erosion of nasal septum. The present study revealed that 1 patient had tubercular infection of maxillary sinus and was 36 years old male patient with past history of pulmonary tuberculosis. There was peripherally enhancing soft tissue mass in left maxillary sinus.64.1\% patients had central nasal septum and 29.5\% had deviated nasal septum, right DNS seen marginally more commonly than left. 'S' shaped deviated septum was seen in only 1 patient. Furthermore, this study also showed 2 cases of frontal osteoma, which were seen in 22 and 56 year old female and male patients respectively.

Both were treated operatively with excision of osteoma. In a study done by Tandon and Mehta et al, 50 cases of osteoma of the paranasal sinuses were studied and the disease was common between 10-30 years age. Most common sinuseswhich were involved were frontal sinuses and then ethmoid sinuses. 2 cases of fibrous dysplasia were seen in the present study in 50 and 23 year old male patients with diffuse involvement of right side maxilla and sphenoid. ${ }^{14}$

Nasal obstruction and swelling are seen in same proportion of patients in both the studies. Nasal discharge is seen less commonly in our study as compared to study by Miyaguchi et al. $80 \%$ of patients also presented with proptosis in this study which was more than in Miyaguchi et al study. Right and left maxillary sinuses were affected equally in malignant lesions of paranasal sinuses. ${ }^{15}$ Extension into surrounding sinuses and nasal cavity is observed in 90\% of patients, followed by extension into orbit and infratemporal fossa. Intracranial and pterygopalatine fossa extension was seen least commonly. Concha bullosa was seen in 15 (19.2\%) patients as the most common anatomical variation in this study. Gwaltney et al found presence of concha bullosa in $35.6 \%$ patients. Inferior turbinate hypertrophy as associated cause for nasal obstruction was seen in 14 (17.9\%) patients in current study followed by $23 \%$ patients in a study conducted by Gwaltney et al study. ${ }^{12}$

\section{CONCLUSION}

Involvement of maxillary and ethmoid sinus is most common in patients of sinusitis who are treated either conservatively or operatively. Most of the patients show either normal or more commonly blocked OMC with only 3 cases showing widening of OMC. $92 \%$ of patients with block OMC did not respond satisfactorily for conservative treatment, as against 75\% patients with normal OMC who responded for conservative treatment. Thus CT scan of paranasal sinuses with axial and coronal reconstruction is the investigation of choice for characterisation, location, extent and diagnosis of PNS pathologies, providing roadmap for surgery and planning of surgery.

\section{REFERENCES}

1. Jones, N.S., CT of the paranasal sinuses: a review of the correlation with clinical, surgical and histopathological findings. Clinical Otolaryngology and Allied Sciences2002; 27(1):11-17.

2. Vikas Dhillon et al. Correlation of Clinical, Radiological and Histopathological Diagnosis among Patients with Sinonasal Masses. International Journal of Contemporary Medical Research. 2016; 3(6):16121615.

3. Selvakumar Subbaraman, Senthilkumar Selvaraj. Fungal Sinonasal Polyposis Causing Unilateral Proptosis. 
International Journal of Contemporary Medical Research. 2016; 4(10):2185-2186.

4. Zinreich S, Albayram S, Benson M, Oliverio P. The ostiomeatal complex and functional endoscopic surgery. In: Som P, ed. Head and neck imaging. 4th ed. St Louis: Mosby, 2003:149-173.

5. Mafee MF, Chow JM, Meyers R: Functional endoscopic sinus surgery: anatomy, CT screening, indications, and complications. Am J Roentgenol 1993; 160(4): 735-44.

6. K. Dua, H. Chopra, AS Khurana, M. Munjal. CT scan variations in chronic sinusitis, Ind J Radiol Imag 2005; 15(3): 315-320.

7. Lohrmann C, Uhl M, Warnatz K, Kotter E, Ghanem N, Langer M.; Sinonasal computed tomography in patients with Wegener's granulomatosis; J Comput Assist Tomogr. 2006; 30(1):122-125.

8. U. Srinivasa Rao, V Sandeep. Clinical and Radiological Study of Antrochoanal Polyps. International Journal of Contemporary Medical Research. 2016; 3(4):11621166.

9. Michaels L. Benign mucosal tumors of the nose and paranasal sinuses. Semin Diagn Pathol 1996; 13(4):113117.

10. J. S. Aswini Jyothi, D. Mounika, M. Vijaya Kumari, N. Jayalatha. CT and MRI in Extracranial Head and Neck Lesions. International Journal of Contemporary Medical Research. 2018; 5 (9):11-19.

11. Shephali S Pawar, Saksham Bansal. CT Anatomy of Paranasal Sinuses - Corelation with Clinical Sinusitis. International Journal of Contemporary Medical Research. 2018; 5(4):1-3.

12. Gwaltney Jr JM, Phillips CD, Miller RD, Riker DK. Computd tomographic study of the common cold. N Eng J Med. 1994; 330(2):25-30.

13. Schramm VL Jr, Inflammatory and neoplastic masses of the nose and paranasal sinus in children. Laryngoscope. 1979; 89(1):1887-97.

14. Tandon PL, Gulati J, Mehta N. Histological study of polypoidal lesions in the nasal cavity. Indian Journal of Otolaryngology and head and neck surgery. 1997; 13(2):3-11.

15. Miyaguchi M, Sakai S, Mori N, Kitaoku S. Symptoms in patients with maxillary sinus carcinoma. J Laryngol Otol. 1990;104 (7):557-559.

16. Batsakis J, El-naggar A, Luna M. Teratoma of head and neck with emphasis on the malignancy. Ann Otol Rhinol Lryngol 1995; 104(5): 496-500.

17. M Miyazaki et al; Simple evaluation of CT findings in the paranasal sinuses for chronic sinusitis; Journal of the Oto Rhino Laryngo Socie of Japan 2000; 103(2): 139146.

18. Andrews AE, Bryson JM, Rowe-Jones JM. Site of origin of nasal polyps: relevance to pathogenesis and management. Rhinology. Sep 2005; 43(3):180-4.

19. JH Wang, YJ Jang, BJ Lee; Natural Course of Retention Cysts of the Maxillary Sinus: Long-Term Follow-Up Results. The Laryngoscope. 2007; 117(2):341-344.

Source of Support: Nil; Conflict of Interest: None

Submitted: 15-11-2018; Accepted: 19-12-2018; Published online: 09-02-2019 